\title{
Assessment of Some Predictors of Weaning from Mechanical Ventilation in Acutely Intoxicated Patients Admitted to the Poison Control Center-Ain Shams University
}

\author{
Asmaa A. Assaf, Heba M. Halawa, Gihan B. Azab, Ahmad F. Younis ${ }^{1}$ and Gamal Eldin M . Elewa ${ }^{2}$ \\ ${ }^{1}$ Department of Forensic Medicine \& Clinical Toxicology \\ ${ }^{2}$ Department of Anesthesia
}

Faculty of Medicine, Ain Shams University, Cairo, Egypt.

\begin{abstract}
:
Background: Respiratory failure is a common complication of poisoning with significant morbidity and mortality. Mechanical ventilation is life saving. Weaning is the process of liberating patient from mechanical ventilatory support. Interleukin-6 (IL-6) and C-reactive protein (CRP) are inflammatory mediators, released in response to many stressful conditions as weaning from mechanical ventilation. Aim of the work: The aim of the study was to assess the role of serum (IL6) level and (CRP) as predictors of outcome of weaning from mechanical ventilation in acutely intoxicated patients admitted to the Poison Control Center- Ain Shams University Hospitals (PCC-ASUHs). And to assess their role in selection of the most appropriate mode for weaning by comparing weaning by pressure support ventilation (PSV) mode, synchronized intermittent mandatory ventilation (SIMV) mode and weaning by the once-daily trial of spontaneous breathing trial by using (T-piece).

Patients and Methods: The study included 58 acutely intoxicated patients, intubated and mechanically ventilated, admitted to the Intensive Care Unit (ICU) of PCC-ASUHs during the period from May 2016 till September 2018. Patients were categorized consecutively according to the outcome of the 2-hour of spontaneous breathing trial (SBT) into 2 groups: Group I (The SBT Success group): included 19 patients who succeed spontaneous breathing trial. Group II (The SBT failed group): included 39 patients who failed SBT which was further subdivided into 3 groups according to the method of weaning: group (1) PSV group, group (2) SIMV group and group (3) Once T-piece group, each group including 13 patients. Results: IL-6 was significantly increased after 2 days in patients who failed the SBT compared to the success group, while CRP was significantly higher in the failed group during weaning trial and 2 days after. There is no significant difference between the three weaning modes regarding IL6 and CRP. Conclusion: Patients who failed the SBT presented higher inflammatory parameters than those who passed. CRP could predict the outcome of weaning from mechanical ventilation.
\end{abstract}

Key words Mechanical ventilation, IL6, CRP.

\section{Introduction}

$\mathrm{M}$ any toxins cause direct respiratory insults that require mechanical ventilation. and opioids come at the top of the list (Bhadra \& Suratt, 2009).

Liberation from mechanical ventilation as early as possible prevents complications associated with prolonged unnecessary mechanical ventilation (Boles et al., 2007).
Significant effort was done to determine and evaluate the possible role of various clinical measurements as predictors for successful weaning (Madani et al., 2013). At present, no single predictor, or set of predictors, has the desired reliability and such a prediction remains a difficult challenge (Verona et al., 2015).

Predictors also have the potential for identifying specific physiological changes responsible for weaning failure (Routsi et al., 2013). 
There is available evidence for weaning-induced cytokine release; Interleukin-6 (IL-6) is one of the proinflammatory cytokines which is generated in response to stress factors such as high inspiratory load (Sellares et al., 2012).

The level of IL-6 in plasma is known as a powerful cause of CRP production in the live. CRP is a plasma protein produced in response to inflammation, infection and stress that measure the acute response to inflammation and is one of the markers of choice in monitoring this response (Black et al., 2004).

Therefore, the aim of this study was to assess the role of systemic interleukin-6 level and C-reactive protein as predictors of outcome of weaning from mechanical ventilation in acutely intoxicated patients admitted to the Poison Control Center- Ain Shams University. Also to assess their role in selection of the most appropriate mode for weaning by comparing weaning by pressure support ventilation(PSV) mode, Synchronized intermittent mandatory ventilation (SIMV) mode and weaning by the once-daily trial of spontaneous breathing method (T-piece).

\section{Subjects and Methods}

\section{I- Subjects}

This work presents a cross sectional prospective study carried out at Poison Control Center of Ain Shams University (PCC-ASU) during the period from May 2016 till September 2018.

\section{Ethical consideration}

After approval from the medical ethical committee, an informed written consent was obtained from each patient or from his/her guardian for inclusion in the study for obtaining medical history, clinical examination, and for the permission to take blood samples for laboratory investigations.

\section{Inclusion criteria}

The selected patients were adults of both sexes, acutely intoxicated, intubated and mechanically ventilated. Patients selected to be similar regarding disease severity and duration of ventilation before weaning.

\section{Exclusion criteria}

- The following patients were excluded:

1.History of respiratory disease.

2.History of cardiac disease.

3.History of renal disease.

4.History of hepatic disease.

5.History of neurological or neuromuscular disease

\section{II- Methods}

Patients were being subjected to 3 step protocol for weaning:

Step 1: (readiness for weaning)

Entry criteria for the weaning trial were as follows:

-Improvement in the underlying condition that led to acute respiratory failure.

-Adequate oxygenation, indicated by $\left.\left(\mathrm{PaO}_{2}\right) / \mathrm{FiO}_{2}\right) \geq 200$, $\mathrm{PaO}_{2} \geq 60 \mathrm{mmHg}, \mathrm{SaO}_{2} \geq 90 \%$ on $\mathrm{FiO}_{2} \leq 0.4$ and PEEP $\leq 5 \mathrm{~cm} \mathrm{H}_{2} \mathrm{O}$.
-Adequate pulmonary function indicated by breath per minute (f) $\leq 35$ and rapid shallow breathing index (RSBI) $\leq 105$ breaths $/ \mathrm{min} / \mathrm{L}$.

- Hemodynamic stability indicated by heart rate less than 140 beats / minute, systolic blood pressure between 90$160 \mathrm{mmHg}$, on no or minimal vasopressors; dopamine < $5 \mu \mathrm{g} / \mathrm{kg}$ per min was be allowed.

-Adequate mental status (arousal, Glasgow Coma Scale score of $\geq 13$, and no continuous sedative infusions).

-Effective cough on a passing suction catheter through the endotracheal tube.

-Core temperature $\leq 38^{\circ} \mathrm{C}$.

-Normal acid base and electrolytes.

Step 2: (spontaneous breathing trial)

When patients met all the above criteria, the next step was to give a short trial of spontaneous breathing by pressure support (received $\mathrm{FiO}_{2} \leq 0.4, \mathrm{PS} \leq 8 \mathrm{cmH}_{2} \mathrm{O}$, and positive end expiratory pressure $\leq 5 \mathrm{cmH}_{2} \mathrm{O}$ for 2 hours).Patients who did not show signs of failure, which were; $\left(\mathrm{SaO}_{2}<90 \%, \mathrm{PaO}_{2}<60 \mathrm{mmHg} ; \mathrm{f}>35\right.$ breaths / minute; HR > 140 beats / minute; systolic BP >180 $\mathrm{mmHg}$ or $<90 \mathrm{mmHg}$; the presence of paradoxical breathing, diaphoresis, agitation, disorientation or depressed mental status), were be extubated.

According to the SBT, patients were categorized consecutively into 2 groups: Group I: included 19 patients who were weaned successfully (The SBT Success group). Group II: included 39 patients who failed weaning (The SBT failed group)

\section{Step 3: (difficult to wean)}

In case of the trial failure (difficult to wean), those patients were classified into three groups (each $n=13$ ). The patients were assigned to the groups in a blinded fashion with the use of opaque, sealed, numbered envelopes, which were opened only when a patient did not successfully complete the two-hour weaning trial:

\section{Group (1) Pressure support Ventilation group (PSV):}

In this group the pressure support was be initially set at $18 \pm 6 \mathrm{cmH}_{2} \mathrm{O}$ with attempting reductions of $2-4 \mathrm{cmH}_{2} \mathrm{O}$ twice a day until $8 \mathrm{cmH}_{2} \mathrm{O}$ could be tolerated.

\section{Group (2) Synchronized intermittent mandatory} ventilation (SIMV):

In this group the initial mandatory breath rate was $10 \pm 2$ breaths per minute with attempting reductions of two to four breaths/min, twice a day, until four breaths/min could be tolerated.

Group (3) Once-daily trial of spontaneous breathing: In this group T-piece trials of increasing duration of spontaneous breathing once-daily were done until $2 \mathrm{~h}$ could be tolerated.

Each patient was subjected for the following:

1)History including:

- Personal data: Age, Sex

- Intoxication data: type of agent, amount, mode, delay time, Presence of co- ingestion

2) Criteria of severity including:

-Acute Physiology And Chronic Health Evaluation II (APACHE II) score calculated during the $1^{\text {st }} 24$ hours. -Duration of ventilation before weaning trial. 
3) Clinical data including:

-Vital data: (Blood pressure, pulse, respiratory rate, core temperature).

-Level of consciousness monitored by Glasgow Coma Scale (GCS).

4) Lung mechanics including:

-Rapid shallow breathing index (RSBI) (f/ $\mathrm{V}_{\mathrm{T}}$, where ' $\mathrm{f}$ ' is the respiratory rate and ' $\mathrm{V}_{\mathrm{T}}$ ' is the tidal volume).

-Minute ventilation (MV).

Measured pre and during weaning trial, patients underwent ventilation for 1 minute by PEEP: 0 and PSV: 0 and then measured.

5) Laboratory investigations: Measured on admission, during and after 2days of weaning trial, including:

-General laboratory investigations: Serum blood glucose, Serum albumin, Liver biochemistry (ALT \& $A S T$ ), Kidney function (serum urea \& creatinine), Electrolytes (Serum sodium, potassium, magnesium \& calcium), Complete blood count, Serial arterial blood gas analysis, CPK-MB.

- Assessment of inflammatory biomarkers:

Serum interleukin-6 level and C-reactive protein.

\section{Statistical analysis}

Data were collected, checked, revised, entered the computer and analyzed by statistical package for statistical analysis (SPSS version 19). Data were presented and analysis was done according to the type of data obtained for each parameter.

\section{I-Descriptive statistics:}

1-Mean, standard deviation $( \pm \mathrm{SD})$ and range were obtained for numerical data.

2-Frequency and percentage were obtained for nonnumerical data.

\section{II- Analytical statistics:}

-Chi-square test: for comparison between two groups with qualitative data.

-Student (t) test: for comparison between two groups with quantitative data.

-One Way ANOVA: for comparison between multiplegroups with quantitative data. Also the Tukey HSD ("Honestly Significant Difference") post-hoc test was used to indicate which groups were significantly different from which others.

The probability $(\mathrm{P})$ was used in all tests. If $\mathrm{P}<0.05$, the comparison was significant (S), If $\mathrm{P}>0.05$, the comparison was non- significant (NS), if $\mathrm{P}<0.01$, the comparison was highly significant.

\section{III-ROC curves}

Receiver Operating Characteristic (ROC) curve determines sensitivity, specificity, and also can determine the significance of a test in predicting certain disease using the Area Under the Curve (AUC) and its significance(Pvalue).

Then, the results of the statistical analysis were represented in tables and histograms for interpretation and discussion.

\section{Results}

Table (1) showed that the highest incidence of poisoning in the studied groups was seen in patients in the middle age group and males were more presented than females. Organophosphate represents the most prevalent poisoning cause for mechanical ventilation in the studied groups.

Regarding vital data and Glasgow Coma Scale (GCS) results in the studied groups, table (2) showed that temperature and respiratory rate (RR) were significantly higher in the failed group compared to the success group pre weaning trial and during weaning trial.

As regards RSBI index there was a highly significant difference between patients who succeeded and who failed weaning trials and also significant difference regarding minute ventilation pre weaning trial and during weaning trial table (3).

As regards $\mathrm{PaO} 2$ and $\mathrm{PaO} 2 / \mathrm{FIO} 2$ were significantly lower in the failed group compared to the success group pre weaning trial and during weaning trial. While $\mathrm{PA}-\mathrm{aO}_{2} \mathrm{G}$ was significantly higher in the failed group as showed in table (4).

As regards Magnesium, Calcium, Hemoglobin, Hct results were significantly lower in the failed group compared to the success group table (5).

As regards results of CPK-MB, IL-6, and CRP levels, table (6) showed that CPK-MB level and IL-6 were significantly increased after 2 days in patients who failed the SBT compared to the success group. While, CRP was significantly higher in the failed group during weaning trial and 2 days after.

By Receiver Operating Characteristic (ROC) curve it was found that Temp, RR, RSBI, Minute ventilation, $\mathrm{PaO}_{2}, \mathrm{PaO}_{2} / \mathrm{FIO}_{2}$ ratio, $\mathrm{PA}-\mathrm{aO}_{2} \mathrm{G}$ and CRP could predict weaning outcome during weaning trial with RSBI and RR had the highest sensitivity and specificity table (7) \& figure (1a,b).

Our results showed that the highest successes rate was in the PSV group (76.9\%), while the highest failure rate was in the SIMV group (38.5\%) as showed in table (8).

Table (9) demonstrated the survival pattern among 39 patients who failed the SBT where 27 cases (69.2\%) survived and the overall mortality was 12 cases $(30.8 \%)$. The cause of mortality in 7 patients as shown was respiratory failure and the shock was the cause of death in the other 5 patients.

There was no significant difference between the three weaning modes regarding IL6 and CRP, 2 days after weaning trial when comparing PSV, SIMV and Once T-piece groups as showed in table (10).

On comparing PSV, SIMV and Once T-piece groups as regards $\mathrm{PO}_{2}, \mathrm{PaO}_{2} / \mathrm{FIO}_{2}$ and $\mathrm{P} \mathrm{A-a} \mathrm{O}_{2} \mathrm{G}$ 2days after weaning trials, there was a significant difference when comparing PSV and SIMV groups and SIMV and Once t- piece groups respectively, while insignificant difference when comparing PSV and once t- piece groups as showed in table (11). 
Table (1) Demographic \& clinical characteristics of different study groups according to SBT Trial.

\begin{tabular}{|c|c|c|}
\hline Characteristics & SBT Success & SBT Failure \\
\hline Number of patients & 19 & 39 \\
\hline $\begin{array}{l}\text { Age; mean } \pm \text { SD } \\
\text { (range) }\end{array}$ & $\begin{array}{c}31 \pm 13.35 \\
(16-52)\end{array}$ & $\begin{array}{c}29.64 \pm 10.66 \\
(16-56)\end{array}$ \\
\hline Gender ratio ;(\%) & $12: 7$ & 27:12 \\
\hline Male: Female & $63.2 \%: 36.8 \%$ & $69.2 \%: 30.8 \%$ \\
\hline \multicolumn{3}{|c|}{ Causes of $M V$} \\
\hline \multicolumn{3}{|c|}{ Drugs of abuse } \\
\hline Opiate & $3(15.8 \%)$ & $7(17.9 \%)$ \\
\hline Tramadol & $3(15.8 \%)$ & $8(20.5 \%)$ \\
\hline \multicolumn{3}{|c|}{ Pesticides } \\
\hline Organophosphate & $5(26.3 \%)$ & $10(25.7 \%)$ \\
\hline Almuninm phosphide & $0(0 \%)$ & $2(5.1 \%)$ \\
\hline \multicolumn{3}{|c|}{ CNS drugs } \\
\hline Clozapine & $2(10.5 \%)$ & $5(12.8 \%)$ \\
\hline TCA & $2(10.5 \%)$ & $2(5.1 \%)$ \\
\hline BNZ & $0(0 \%)$ & $1(2.6 \%)$ \\
\hline Baclofen & $0(0 \%)$ & $1(2.6 \%)$ \\
\hline \multicolumn{3}{|c|}{ Others } \\
\hline Beta blockers & $0(0 \%)$ & $2(5.1 \%)$ \\
\hline Methanol & $1(5.3 \%)$ & $1(2.6 \%)$ \\
\hline $\mathrm{CO}$ & $3(15.8 \%)$ & $0(0 \%)$ \\
\hline \multicolumn{3}{|c|}{ Criteria of severity } \\
\hline APACHE II Score & $20.58 \pm 5.55$ & $20.18 \pm 3.22$ \\
\hline $\begin{array}{c}\text { Mechanicalventilation days } \\
\text { before weaning trial }\end{array}$ & $3.47 \pm 1.04$ & $3.56 \pm 0.93$ \\
\hline
\end{tabular}

Abbreviations: MV: mechanical ventilation; TCA: tricyclic antidepresent; BNZ: benzodiazepine; CO:carbone monoxide; h: hour; APACHE II: Acute Physiological Assessment and Chronic Health Evaluation II score. Data are presented as mean $\pm S D$ or $\%$.

Table (2) Independent t-test showing comparison between the studied groups as regards vital signs\&GCS.

\begin{tabular}{|c|c|c|c|c|c|c|c|c|c|}
\hline \multirow[t]{2}{*}{ Variable } & \multicolumn{3}{|c|}{ On admission } & \multicolumn{3}{|c|}{ Pre weaning trial } & \multicolumn{3}{|c|}{ During weaning trial } \\
\hline & $\begin{array}{c}\text { SBT } \\
\text { Success } \\
=19)\end{array}$ & $\begin{array}{c}\begin{array}{c}\text { SBT } \\
\text { failure } \\
(\mathrm{n}=39)\end{array} \\
\end{array}$ & $P$ value & $\begin{array}{c}\text { SBT } \\
\text { success } \\
(\mathrm{n}=19) \\
\end{array}$ & $\begin{array}{l}\text { SBT } \\
\text { Failure } \\
(\mathrm{n}=39)\end{array}$ & $P$ value & $\begin{array}{c}\text { SBT } \\
\text { success } \\
(\mathrm{n}=19)\end{array}$ & $\begin{array}{l}\text { SBT } \\
\text { failure } \\
(\mathrm{n}=39)\end{array}$ & $P$ value \\
\hline $\begin{array}{ll}\text { Mean } & \text { arterial } \\
\text { pressure }\end{array}$ & $\begin{array}{l}74.74 \pm \\
17.86\end{array}$ & $\begin{array}{l}81.35 \pm \\
15.75\end{array}$ & 0.1567 & $\begin{array}{l}96.53 \pm \\
7.60\end{array}$ & $\begin{array}{l}96.85 \pm \\
12.78\end{array}$ & 0.9203 & $\begin{array}{l}100.11 \pm \\
11.53\end{array}$ & $\begin{array}{l}103 \pm \\
14.26\end{array}$ & 4455 \\
\hline Heart rate & $\begin{array}{l}107.68 \pm \\
20.34\end{array}$ & $\begin{array}{l}99.44 \pm \\
23.75\end{array}$ & 0.2000 & $\begin{array}{l}104.89 \pm \\
14.81\end{array}$ & $\begin{array}{l}107 \pm \\
15.09\end{array}$ & 0.6171 & $\begin{array}{l}110.11 \pm \\
15.62\end{array}$ & $\begin{array}{l}117.31 \pm \\
16.26\end{array}$ & 1146 \\
\hline Temperature & $\begin{array}{l}37.21 \pm \\
0.34\end{array}$ & $\begin{array}{l}37.45 \pm \\
0.63 \\
\end{array}$ & 0.1269 & $\begin{array}{l}37.58 \pm \\
0.38\end{array}$ & $\begin{array}{l}37.79 \pm \\
0.31 \\
\end{array}$ & $\begin{array}{c}0.0286 \\
(\mathrm{~S})\end{array}$ & $\begin{array}{l}37.67 \pm \\
0.34 \\
\end{array}$ & $\begin{array}{l}37.94 \pm \\
0.34\end{array}$ & $\begin{array}{l}0.0063 \\
(\mathrm{~S})\end{array}$ \\
\hline$\overline{R R}$ & $\begin{array}{l}20.35 \pm \\
12.02\end{array}$ & $\begin{array}{l}25.08 \pm \\
7.82\end{array}$ & 0.0768 & $\begin{array}{l}23.26 \pm \\
2.38\end{array}$ & $\begin{array}{l}28.26 \pm \\
3.14\end{array}$ & $\begin{array}{c}0.0001 \\
(\mathrm{~S})\end{array}$ & $\begin{array}{l}27.47 \pm \\
2.58 \\
\end{array}$ & $\begin{array}{l}36.56 \pm \\
2.35\end{array}$ & $\begin{array}{l}0.0001 \\
(\mathrm{~S})\end{array}$ \\
\hline $\begin{array}{l}\text { Glasgow Coma } \\
\text { Scale (GCS). }\end{array}$ & $\begin{array}{l}6.53 \pm \\
3.70\end{array}$ & $\begin{array}{l}5.31 \pm \\
2.15\end{array}$ & 0.1178 & $\begin{array}{l}13.95 \pm \\
0.69\end{array}$ & $\begin{array}{l}13.79 \pm \\
0.82\end{array}$ & 0.4668 & $\begin{array}{l}12.74 \pm \\
3.75\end{array}$ & $\begin{array}{l}13.41 \pm \\
0.81\end{array}$ & 0.2871 \\
\hline
\end{tabular}

Abbreviations: RR:respiratory rate; GCS: Glasgow Coma Scale. Data are presented as mean \pm SD. S :Significant

Table (3) Independent t-test showing comparison between the studied groups as regards RBSI and Minute ventilation.

\begin{tabular}{|l|c|c|c|c|c|c|}
\hline \multicolumn{1}{|c|}{ Variable } & \multicolumn{3}{|l|}{ Pre weaning trial } & \multicolumn{3}{l|}{ During weaning trial } \\
\hline & $\begin{array}{c}\text { SBTsuccess } \\
(\mathrm{n}=19)\end{array}$ & $\begin{array}{c}\text { SBT failure } \\
(\mathrm{n}=39)\end{array}$ & $\mathrm{P}$ value & $\begin{array}{c}\text { SBT success } \\
(\mathrm{n}=19)\end{array}$ & $\begin{array}{c}\text { SBT failure } \\
(\mathrm{n}=39)\end{array}$ & P value \\
\hline $\begin{array}{l}\text { RSBI } \\
\text { (BREATHS/MIN/L) }\end{array}$ & $42.11 \pm 12.17$ & $76.44 \pm 15.07$ & $0.0001(\mathrm{~S})$ & $53.63 \pm 10.9$ & $105.21 \pm 20.10$ & $0.0001(\mathrm{~S})$ \\
\hline $\begin{array}{l}\text { Minute ventilation } \\
(\mathrm{L} / \mathrm{MIN})\end{array}$ & $13.46 \pm 1.37$ & $12.00 \pm 1.92$ & $0.0045(\mathrm{~S})$ & $13.94 \pm 1.18$ & $12.82 \pm 2.10$ & $0.0352(\mathrm{~S})$ \\
\hline
\end{tabular}

Abbreviations: RSBI: rapid shallow breathing index. Data are presented as mean $\pm S D . S$ :Significant 
Table (4) Independent t-test showing comparison between groups regaring ABG, PaO2/FIO2 \& A-aO2 G.

\begin{tabular}{|c|c|c|c|c|c|c|c|c|c|}
\hline \multirow[t]{2}{*}{ Variable } & \multicolumn{3}{|c|}{ On admission } & \multicolumn{3}{|c|}{ Pre weaning trial } & \multicolumn{3}{|c|}{ During weaning trial } \\
\hline & $\begin{array}{c}\text { SBT } \\
\text { success } \\
(n=19)\end{array}$ & $\begin{array}{c}\text { SBT } \\
\text { failure } \\
(\mathrm{n}=39)\end{array}$ & P value & $\begin{array}{c}\text { SBT } \\
\text { success } \\
(n=19)\end{array}$ & $\begin{array}{c}\text { SBT } \\
\text { failure } \\
(n=39)\end{array}$ & $\mathrm{P}$ value & $\begin{array}{c}\text { SBT } \\
\text { success } \\
(\mathrm{n}=19)\end{array}$ & $\begin{array}{c}\text { SBT } \\
\text { failure } \\
(\mathrm{n}=39)\end{array}$ & $\mathrm{P}$ value \\
\hline $\mathrm{pH}$ & $\begin{array}{l}7.27 \pm \\
0.11\end{array}$ & $\begin{array}{l}7.29 \pm \\
0.14\end{array}$ & 0.5877 & $\begin{array}{l}7.43 \pm \\
0.05\end{array}$ & $\begin{array}{l}7.45 \pm \\
0.06\end{array}$ & 0.2148 & $\begin{array}{l}7.39 \pm \\
0.05\end{array}$ & $\begin{array}{l}7.40 \pm \\
0.08\end{array}$ & 0.6203 \\
\hline $\begin{array}{l}\mathrm{HCO} 3 \\
(\mathrm{mEq} / \mathrm{L})\end{array}$ & $\begin{array}{c}21.83 \pm \\
4.45\end{array}$ & $\begin{array}{c}20.24 \pm \\
5.59\end{array}$ & 0.2837 & $\begin{array}{c}25.18 \pm \\
3.69\end{array}$ & $\begin{array}{c}23.97 \pm \\
4.95\end{array}$ & 0.3494 & $\begin{array}{c}25.86 \pm \\
3.67\end{array}$ & $\begin{array}{c}24.47 \pm \\
5.78\end{array}$ & 0.3431 \\
\hline $\begin{array}{l}\mathrm{PaO} 2 \\
(\mathrm{~mm} \mathrm{Hg})\end{array}$ & $\begin{array}{l}55.95 \pm \\
7.96\end{array}$ & $\begin{array}{c}57.00 \pm \\
8.97\end{array}$ & 0.6663 & $\begin{array}{c}124.05 \pm \\
15.67\end{array}$ & $\begin{array}{l}99.03 \pm \\
8.75\end{array}$ & $\begin{array}{l}0.0001 \\
(\mathrm{~S})\end{array}$ & $\begin{array}{c}116.05 \pm \\
14.76\end{array}$ & $\begin{array}{c}87.87 \pm \\
8.08\end{array}$ & $\begin{array}{l}0.0001 \\
(\mathrm{~S})\end{array}$ \\
\hline $\begin{array}{l}\mathrm{PaCO} 2 \\
(\mathrm{~mm} \mathrm{Hg})\end{array}$ & $\begin{array}{c}49.63 \pm \\
13.70\end{array}$ & $\begin{array}{c}42.81 \pm \\
15.4\end{array}$ & 0.1069 & $\begin{array}{c}38.95 \pm \\
6.05\end{array}$ & $\begin{array}{c}36.22 \pm \\
6.48\end{array}$ & 0.1297 & $\begin{array}{c}42.05 \pm \\
5.70\end{array}$ & $\begin{array}{c}39.97 \pm \\
7.18\end{array}$ & 0.2747 \\
\hline $\mathrm{BD}$ & $\begin{array}{c}-4.38 \pm \\
4.75\end{array}$ & $\begin{array}{c}-6.16 \pm \\
6.32\end{array}$ & 0.2824 & $\begin{array}{l}1.79 \pm \\
3.43\end{array}$ & $\begin{array}{c}-0.04 \pm \\
4.88\end{array}$ & 0.1486 & $\begin{array}{c}1.16 \pm \\
3.61\end{array}$ & $\begin{array}{c}-0.27 \pm \\
6.07\end{array}$ & 0.3482 \\
\hline $\mathrm{PaO} 2 / \mathrm{FIO} 2$ & $\begin{array}{l}266 \pm \\
37.82\end{array}$ & $\begin{array}{c}270.56 \pm \\
42.50\end{array}$ & 0.6929 & $\begin{array}{c}309.79 \pm \\
39.19\end{array}$ & $\begin{array}{c}247.36 \pm \\
21.85\end{array}$ & $\begin{array}{l}0.0001 \\
(\mathrm{~S})\end{array}$ & $\begin{array}{c}289.89 \pm \\
36.91\end{array}$ & $\begin{array}{c}218.92 \pm \\
21.85\end{array}$ & $\begin{array}{l}0.0001 \\
(\mathrm{~S})\end{array}$ \\
\hline $\begin{array}{c}\text { PA-aO2 G } \\
(\mathrm{mm} \mathrm{Hg})\end{array}$ & $\begin{array}{c}32.69 \pm \\
15.61\end{array}$ & $\begin{array}{c}39.32 \pm \\
17.59\end{array}$ & 0.1683 & $\begin{array}{c}113.03 \pm \\
12.60\end{array}$ & $\begin{array}{c}143.67 \pm \\
10.04\end{array}$ & $\begin{array}{l}0.0001 \\
(\mathrm{~S})\end{array}$ & $\begin{array}{c}116.56 \pm \\
11.96\end{array}$ & $\begin{array}{c}147.35 \pm \\
10.23\end{array}$ & $\begin{array}{c}0.0001 \\
\text { (S) }\end{array}$ \\
\hline
\end{tabular}

Abbreviations: $\mathrm{HCO}_{3}$ : serum bicarbonate; $\mathrm{PaO}_{2}$ : arterial partial pressure of oxygen; $\mathrm{PaCO}$ : arterial partial pressure of carbon dioxide; $\mathrm{BD}$ : base deficit; $\mathrm{PaO}_{2} / \mathrm{FiO}_{2}$ ratio: ratio of arterial oxygen tension to fraction of inspired oxygen; A$\mathrm{aO}_{2} \mathrm{G}$ : alveolar-arterial $\mathrm{O}_{2}$ gradient .Data are presented as mean $\pm S D$. S :Significant.

Table (5) Independent t-test showing comparison between groups as regards serum electrolytes \& CBC.

\begin{tabular}{|c|c|c|c|c|c|c|c|c|c|}
\hline \multirow[t]{2}{*}{ Variable } & \multicolumn{3}{|c|}{ On admission } & \multicolumn{3}{|c|}{ during weaning trial } & \multicolumn{3}{|c|}{ 2days after weaning trial } \\
\hline & $\begin{array}{c}\text { SBT } \\
\text { success } \\
(n=19)\end{array}$ & $\begin{array}{c}\text { SBT } \\
\text { failure } \\
(n=39)\end{array}$ & $\begin{array}{c}\mathrm{P} \\
\text { value }\end{array}$ & $\begin{array}{c}\text { SBT } \\
\text { success } \\
(n=19)\end{array}$ & $\begin{array}{c}\text { SBT } \\
\text { failure } \\
(\mathrm{n}=39)\end{array}$ & $\begin{array}{c}\mathrm{P} \\
\text { value }\end{array}$ & $\begin{array}{c}\text { SBT } \\
\text { success } \\
(n=19)\end{array}$ & $\begin{array}{c}\text { SBT } \\
\text { failure } \\
(n=39)\end{array}$ & $\begin{array}{c}\mathrm{P} \\
\text { value }\end{array}$ \\
\hline $\begin{array}{l}\text { Electrolytes: } \\
\text { Sodium } \\
(\mathrm{mg} / \mathrm{dL})\end{array}$ & $\begin{array}{c}132.11 \pm \\
6.98\end{array}$ & $\begin{array}{c}134.85 \pm \\
5.33\end{array}$ & 0.1031 & $\begin{array}{c}134.21 \pm \\
4.10\end{array}$ & $\begin{array}{c}35.95 \pm \\
5.74\end{array}$ & 0.2428 & $\begin{array}{c}135.21 \pm \\
3.91\end{array}$ & $\begin{array}{c}136.31 \pm \\
4.79\end{array}$ & 0.3887 \\
\hline $\begin{array}{l}\text { Potasium } \\
(\mathrm{mg} / \mathrm{dL})\end{array}$ & $\begin{array}{c}3.66 \pm \\
0.64\end{array}$ & $\begin{array}{c}3.54 \pm \\
0.50\end{array}$ & 0.4379 & $\begin{array}{c}3.65 \pm \\
0.63\end{array}$ & $\begin{array}{c}3.52 \pm \\
0.67\end{array}$ & 0.4826 & $\begin{array}{c}3.29 \pm \\
0.41\end{array}$ & $\begin{array}{c}3.48 \pm \\
0.63\end{array}$ & 0.2374 \\
\hline Magnesium(mg/dL) & $\begin{array}{c}2.19 \pm \\
0.23\end{array}$ & $\begin{array}{c}2.01 \pm \\
0.31\end{array}$ & $\begin{array}{l}0.028 \\
(\mathrm{~S})\end{array}$ & $\begin{array}{c}2.09 \pm \\
0.23\end{array}$ & $\begin{array}{c}1.88 \pm \\
0.31\end{array}$ & $\begin{array}{l}0.0114 \\
(\mathrm{~S})\end{array}$ & $\begin{array}{c}1.98 \pm \\
0.20\end{array}$ & $\begin{array}{c}1.78 \pm \\
0.31\end{array}$ & $\begin{array}{l}0.0132 \\
(\mathrm{~S})\end{array}$ \\
\hline Calcium(mg/dL) & $\begin{array}{c}9.18 \pm \\
0.38\end{array}$ & $\begin{array}{l}8.89 \pm \\
0.55\end{array}$ & $\begin{array}{l}0.0434 \\
(\mathrm{~S})\end{array}$ & $\begin{array}{c}9.07 \pm \\
0.42\end{array}$ & $\begin{array}{c}8.67 \pm \\
0.53\end{array}$ & $\begin{array}{l}0.0001 \\
(\mathrm{~S})\end{array}$ & $\begin{array}{l}8.99 \pm \\
0.44\end{array}$ & $\begin{array}{l}8.52 \pm \\
0.53\end{array}$ & $\begin{array}{l}0.0015 \\
(\mathrm{~S})\end{array}$ \\
\hline $\begin{array}{c}\text { CBC: } \\
\text { Hemoglobin(g/dL) }\end{array}$ & $\begin{array}{c}2.92 \pm \\
1.39\end{array}$ & $\begin{array}{l}12.14 \pm \\
1.76\end{array}$ & 0.0967 & $\begin{array}{c}2.25 \pm \\
1.58\end{array}$ & $\begin{array}{c}1.16 \pm \\
1.79\end{array}$ & $\begin{array}{l}0.0278 \\
(\mathrm{~S})\end{array}$ & $\begin{array}{c}11.6 \pm \\
1.64\end{array}$ & $\begin{array}{c}10.58 \pm \\
1.80\end{array}$ & $\begin{array}{l}0.0418 \\
(\mathrm{~S})\end{array}$ \\
\hline Hct $(\%)$ & $\begin{array}{c}40.78 \pm \\
5.84\end{array}$ & $\begin{array}{l}37.65 \pm \\
6.28\end{array}$ & 0.0739 & $\begin{array}{c}9.91 \pm \\
5.19\end{array}$ & $\begin{array}{c}34.92 \pm \\
6.33\end{array}$ & $\begin{array}{l}0.0043 \\
(\mathrm{~S})\end{array}$ & $\begin{array}{c}38.53 \pm \\
6.74\end{array}$ & $\begin{array}{c}34.12 \pm \\
6.13\end{array}$ & $\begin{array}{l}0.0158 \\
(\mathrm{~S})\end{array}$ \\
\hline $\begin{array}{c}\text { TLC } \\
\left(10^{\wedge} 3 / \mu \mathrm{L}\right)\end{array}$ & $\begin{array}{c}12.43 \pm \\
4.60\end{array}$ & $\begin{array}{l}12.23 \pm \\
4.83\end{array}$ & 0.8811 & $\begin{array}{l}12.82 \pm \\
5.37\end{array}$ & $\begin{array}{c}14.29 \pm \\
6.23\end{array}$ & 0.3823 & $\begin{array}{l}11.77 \pm \\
4.63\end{array}$ & $\begin{array}{c}\mathrm{M} \\
13.17 \pm 7.47\end{array}$ & 0.4576 \\
\hline $\begin{array}{c}\mathrm{PL} \\
\left(10^{\wedge} 3 / \mu \mathrm{L}\right)\end{array}$ & $\begin{array}{c}227.05 \pm \\
55.19\end{array}$ & $\begin{array}{c}209.64 \pm \\
84.42\end{array}$ & 0.4179 & $\begin{array}{c}234.21 \pm \\
62.66\end{array}$ & $\begin{array}{c}206.41 \pm \\
79.64\end{array}$ & 0.1883 & $\begin{array}{c}227.37 \pm \\
70.03\end{array}$ & $\begin{array}{c}208.90 \pm \\
94.56\end{array}$ & 0.4534 \\
\hline
\end{tabular}

Hct: hematocrite; TLC: total leucocytic count; PL:platlat count. Data are presented as mean \pm SD. S :Significant

Table (6) Independent t-test showing comparison between the studied groups as regards CPK-MB, Serum interleukin-6 level, C-reactive protein.

\begin{tabular}{|l|c|c|c|c|c|l|c|c|c|}
\hline Variable & \multicolumn{2}{|l|}{ On admission } & \multicolumn{2}{|c|}{ During weaning trial } & \multicolumn{3}{|c|}{ 2 days after weaning trial } \\
\hline & $\begin{array}{c}\text { SBT } \\
\text { success } \\
(\mathrm{n}=19)\end{array}$ & $\begin{array}{c}\text { SBT } \\
\text { failure } \\
(\mathrm{n}=39)\end{array}$ & P value & $\begin{array}{c}\text { SBT } \\
\text { success } \\
(\mathrm{n}=19)\end{array}$ & $\begin{array}{c}\text { SBT } \\
\text { failure } \\
(\mathrm{n}=39)\end{array}$ & P value & $\begin{array}{c}\text { SBT } \\
\text { success } \\
(\mathrm{n}=19)\end{array}$ & $\begin{array}{c}\text { SBT } \\
\text { failure } \\
(\mathrm{n}=39)\end{array}$ & P value \\
\hline CPK-MB & $22.58 \pm$ & $25.10 \pm 13.85$ & 0.49 & $24.32 \pm$ & $31.15 \pm$ & 0.1946 & $20.32 \pm$ & $30.10 \pm$ & 0.0258 \\
$(\mathrm{U} / \mathrm{L})$ & 11.78 & & 11.24 & 21.21 & & 8.49 & 17.58 & $(\mathrm{~S})$ \\
\hline IL-6 & $44.16 \pm$ & $46.56 \pm$ & 0.73 & $40.37 \pm$ & $50.62 \pm$ & 0.0975 & $24.05 \pm$ & $40.41 \pm$ & 0.0149 \\
$(\mathrm{ng} / \mathrm{L})$ & 22.76 & 26.22 & & 12.82 & 24.87 & & 6.14 & 27.94 & $(\mathrm{~S})$ \\
\hline $\mathrm{CRP}$ & $31 \pm$ & $43.13 \pm$ & 0.1048 & $25.63 \pm$ & $39.77 \pm$ & 0.0050 & $21.53 \pm$ & $37.72 \pm$ & 0.0146 \\
$(\mathrm{mg} / \mathrm{L})$ & 19.17 & 29.07 & & 13.12 & 18.94 & $(\mathrm{~S})$ & 17.13 & 25.26 & $(\mathrm{~S})$ \\
\hline
\end{tabular}

Abbreviations: CPK-MB: Serum creatine kinase-MB; IL6: interleukin 6; CRP: C-reactive protein. Data are presented as mean $\pm S D$. S: Significant. 
Table (7) Sensitivity and specificity of significant parameters measured during weaning trial for prediction of weaning outcome.

\begin{tabular}{|l|l|l|l|l|l|l|l|l|}
\hline Parameters & AUC & P & Cut-off & Sensitivity & Specificity & PPV & NPV & Total accuracy \\
\hline Temperature & 0.686 & 0.022 & 37.9 & 56.41 & 100.00 & 100.00 & 52.78 & 70.69 \\
\hline RR & 0.988 & $<0.0001$ & 33 & 97.44 & 100.00 & 100.00 & 95.00 & 98.28 \\
\hline RSBI & 0.999 & $<0.0001$ & 74 & 97.44 & 100.00 & 100.00 & 95.00 & 98.28 \\
\hline Minute ventilation & 0.682 & 0.025 & 11.45 & 74.36 & 100.00 & 100.00 & 65.52 & 82.76 \\
\hline $\mathrm{PaO} 2$ & 0.955 & $<0.0001$ & 99 & 92.31 & 89.47 & 94.74 & 85.00 & 91.38 \\
\hline $\mathrm{PaO} 2 /$ FIO2 & 0.955 & $<0.0001$ & 247 & 92.31 & 89.47 & 94.74 & 85.00 & 91.38 \\
\hline PA-aO2 & 0.957 & $<0.0001$ & 137 & 87.18 & 100.00 & 100.00 & 79.17 & 91.38 \\
\hline CRP & 0.666 & 0.042 & 37 & 58.97 & 89.47 & 92.00 & 51.52 & 68.97 \\
\hline
\end{tabular}

RR: respiratory rate; $\mathrm{RSBI}$ : rapid shallow breathing index; $\mathrm{PaO}_{2}$ : arterial partial pressure of oxygen; $\mathrm{PaO}_{2} / \mathrm{FiO}_{2}$ ratio: ratio of arterial oxygen tension to fraction of inspired oxygen; $\mathrm{PA}-a \mathrm{O}_{2}$ G: alveolararterial O2 gradient; CRP:C-reactive protein; AUC: Area under Curve; PPV: Positive Predictive Value; NPV: Negative Predictive Value.

Table (8) Comparison between the three modes of weaning as regards weaning outcome.

\begin{tabular}{|c|c|c|c|c|c|c|}
\hline \multirow{3}{*}{ Weaning outcome } & \multicolumn{6}{|c|}{ The studied groups } \\
\hline & \multicolumn{2}{|c|}{$\begin{array}{l}\text { PSV } \\
N=13\end{array}$} & \multicolumn{2}{|c|}{$\begin{array}{l}\text { SIMV } \\
N=13\end{array}$} & \multicolumn{2}{|c|}{$\begin{array}{l}\text { Once } \\
N=13\end{array}$} \\
\hline & No & $\%$ & No & $\%$ & No & $\%$ \\
\hline Final Success & 10 & $76.9 \%$ & 8 & $61.5 \%$ & 9 & $69.2 \%$ \\
\hline Final Failure & 3 & $23.1 \%$ & 5 & $38.5 \%$ & 4 & $30.8 \%$ \\
\hline
\end{tabular}

Table (9) Survival outcome and causes of death among 39 patients who failed the SBT.

\begin{tabular}{|c|c|c|c|c|}
\hline Outcome & Survival & Death & & Total \\
\hline Cause of death & & Respiratory failure & Shock & \\
\hline Number & 27 & 7 & 5 & 39 \\
\hline Percentage & 69.2 & 18 & 12.8 & 100 \\
\hline
\end{tabular}

Table (10) ANOVA One-way analysis comparing between the three groups as regards IL6 and Creactive protein 2 days after weaning trials.

\begin{tabular}{|l|l|l|l|c|}
\hline \multirow{2}{*}{ Variable } & The studied groups & $P$ value \\
\cline { 2 - 5 } & PSV & SIMV & Once & T-piece \\
& $N=13$ & $N=13$ & $5=13$ & 0.2508 \\
\hline IL-6 (ng/L) & $52.85 \pm 24.17$ & $57.15 \pm 25.59$ & $51.23 \pm 24.52$ & 0.4042 \\
\hline CRP (mg/L) & $57.62 \pm 22.5$ & $64.23 \pm 26.79$ & $51.31 \pm 22.98$ & \\
\hline
\end{tabular}

Abbreviations: PSV:pressure support ventilation; SIMV : synchronous intermittent mandatory ventilation; Once Tpiece: Once-daily trial of spontaneous breathing using t-tube; IL6:interleukin 6; CRP:C-reactive protein.

Table (11) ANOVA one way statistical analysis, comparing PSV and SIMV, and once t- piece groups as regards PO2, PaO2/FIO2 and $\mathrm{P}$ A-a O2 G 2days after weaning trials.

\begin{tabular}{|c|c|c|c|c|c|}
\hline \multirow{2}{*}{ Variable } & \multicolumn{3}{|c|}{ The studied groups } & \multirow{2}{*}{ ANOVA } & \multirow{2}{*}{ TUKEY'S TEST } \\
\hline & $\operatorname{PSV} N=13$ & SIMV $N=13$ & OnceT-piece $N=13$ & & \\
\hline $\begin{array}{c}\mathrm{PaO} 2 \\
(\mathrm{~mm} \mathrm{Hg})\end{array}$ & $105.18 \pm 7.26$ & $97 \pm 7.60$ & $107.19 \pm 10.34$ & $0.0102(\mathrm{~S})$ & $\begin{array}{c}\mathrm{P} 1=0.0494(\mathrm{~S}) \\
\mathrm{P} 2=0.8199 \\
\mathrm{P} 3=0.0116(\mathrm{~S})\end{array}$ \\
\hline $\mathrm{PaO} 2 / \mathrm{FIO} 2$ & $264.85 \pm 25.64$ & $242.38 \pm 19.02$ & $267.85 \pm 24.80$ & $0.0163(\mathrm{~S})$ & $\begin{array}{c}\mathrm{P} 1=0.0489(\mathrm{~S}) \\
\mathrm{P} 2=0.9426 \\
\mathrm{P} 3=0.0227(\mathrm{~S})\end{array}$ \\
\hline $\begin{array}{c}\text { PA-aO2 G } \\
(\mathrm{mm} \mathrm{Hg})\end{array}$ & $130.24 \pm 11.47$ & $142.19 \pm 12.72$ & $130.89 \pm 10.77$ & $0.0211(\mathrm{~S})$ & $\begin{array}{c}\mathrm{P} 1=0.0344(\mathrm{~S}) \\
\mathrm{P} 2=0.9890 \\
\mathrm{P} 3=0.0476(\mathrm{~S})\end{array}$ \\
\hline
\end{tabular}

Abbreviations: PSV: pressure support ventilation; SIMV: synchronous intermittent mandatory ventilation; Once Tpiece: Once-daily trial of spontaneous breathing using t-tube; $\mathrm{PaO}_{2}$ : arterial partial pressure of oxygen; $\mathrm{PaO}_{2} / \mathrm{FiO}_{2}$ ratio: ratio of arterial oxygen tension to fraction of inspired oxygen; $P A-a O_{2} \quad G$ : alveolar-arterial $\mathrm{O}_{2}$ gradient. $S$ : Significant. Tukey HSD Post-hoc Test...P $<0.05=$ significant at $95 \%$ confidence intervals $(C I): P 1=P S V$ vs SIMV groups. $P 2=P S V$ vs $t$ piece groups.P3 $=$ SIMV vs $t$ piece groups. 

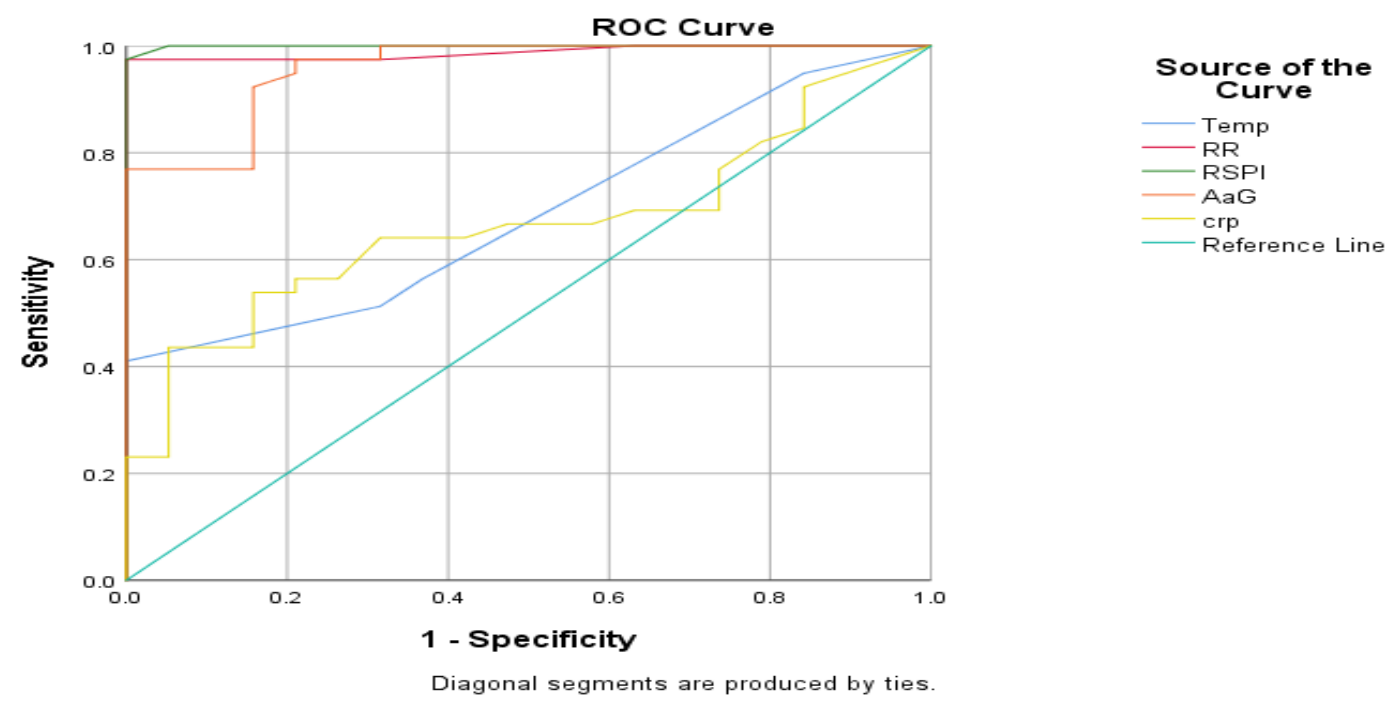

(a)
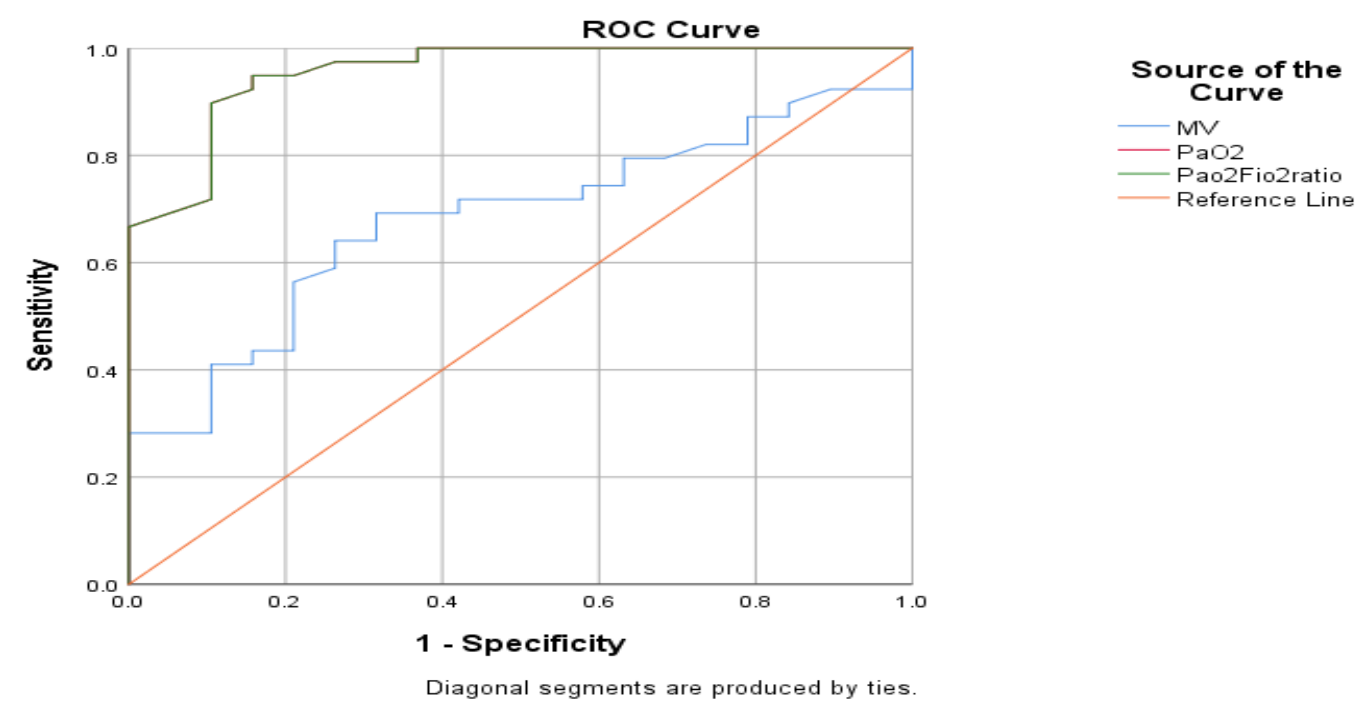

(b)

Figure (1a,b): Receiver Operating Characteristic (ROC) curves of significant parameters measured during weaning trial for prediction of weaning outcome (a): Temp, RR, RSBI, A-aG, CRP \& (b): Minute ventilation, $\mathrm{PO}_{2}, \mathrm{PO}_{2} / \mathrm{FIO}_{2}$ ratio.

\section{Discussion}

The process of weaning may impose an increased load on respiratory muscles. High inspiratory load results in increased mechanical stress, increases permeability and induces inflammation with increased in IL-6 levels, which could stimulate increased CRP, reflecting the intensity of the inflammation process (Sellares et al., 2012).

The present study included fifty eight acutely intoxicated patients, intubated and mechanically ventilated, in the ICU of PCC of Ain Shams University during the period from May 2016 till September 2018.

It was noticed that the highest incidence of poisoning in the studied groups was seen in patients in the middle age group which could be explained by the increased stress conditions that can happen during this age. This was similar to the study done by Ahmed et al.,2014 that included 117 ICU patients with acute OP poisoning requiring mechanical ventilation.

Males were more affected than females, the possible explanation of male predominance in the current study is the liability of males to life stresses and strains in our society because of unemployment, poverty and depression. This was also seen in the study of El Maraghiet al., 2014 and Georgakaset al., 2018 which males were more affected than females.

The present study showed that organophosphate represent the most prevalent poisoning requiring mechanical ventilation in both the SBT success group and the SBT failed group with percentage $26.3 \%, 25.7 \%$ respectively. The possible explanation of this predominance is the easy availability of insecticides as household products. Previously, Nazima et al., 2018 reported that the total poisoning admissions during their study period from August 2006 to August 2010 were 2057, among them 1258 were organophosphorous poisoning cases which constituted $61.16 \%$ of total poisoning admissions among them 254 needed mechanical ventilation. 
The present study showed that temperature and respiratory rate $(\mathrm{RR})$ were significantly higher in the failed group compared to the success group.

Higher temperature increases peripheral oxygen utilization leading to decrease mixed venous oxygen content and increase carbon dioxide production which increase ventilatory demand predisposing to failure of SBT (Epstein, 2009).

On the contrary, Yoo et al., 2018 that carried out on sixty patients subjected to extubation trial in the medical or surgical intensive care unit from May 2015 through February 2016, showed that there was statistically non-significant difference between both groups regarding temperature.

In the study of Spadaro et al., 2016 that carried out on 51 ready-to-wean patients. The mean RR was significantly higher in the failed group compared to the success group. On the contrary, other studies showed that there was statistically non-significant difference between both groups regarding RR (Monaco et al., 2010).

The present study showed that RR could predict weaning outcome during weaning trial, it was found that RR at cut-off point 33 breath/min had corresponding sensitivity $97.44 \%$ and specificity 100 $\%$. In the study conducted by Lima, 2013 RR was also an effective predictor of weaning failure. The best cutoff point was RR > $24 \mathrm{rpm}$, sensitivity $100 \%$, specificity $85 \%$, and accuracy $88 \%$ which differed from those reported in the literature suggesting that the cut-off points of 35 and 38 are too high for our reality.

Regarding lung mechanics, the present study, found that RSBI showed highly significant decrease in success group compared to failed group pre-weaning trial and during weaning trial. Also, the result showed that RSBI, can predict weaning outcome during weaning trial, it was found that RSBI at cut-off 74 breaths/min/l had corresponding sensitivity of $97.44 \%$ and specificity of $100 \%$.

In accordance, Goncalves et al., 2017 in their prospective study which conducted on 40 patients where RSBI was measured in three different methods of weaning, it was found the RSBI with the cutoff point of $78 \mathrm{cycles} / \mathrm{min} / \mathrm{L}$ is the best strategy to identify patients that are ready for MV withdrawal.

RSBI has been the most often employed index to evaluate MV withdrawal. Currently, some institutions rely exclusively on the value of RSBI measured once a day before submitting the patient to SBT or extubation.

As regards minute ventilation the present study results showed that minute ventilation was lower in failed group in comparison to succeed group preweaning trial and during weaning trial.

This finding was in agreement with the prospective study done by Okamoto et al.,1990 on 25 patients who required mechanical ventilatory support after major surgery or severe burns, the result showed that minute ventilation was lower in failed group in comparison to succeed group.

Low Minute ventilation may arise from decreased respiratory center drive, a structural abnormality of the lungs or of the thoracic cage, or respiratory muscle dysfunction (Kallet, 2011).

$\mathrm{PaO}_{2}$ and $\mathrm{PaO}_{2} / \mathrm{FIO}_{2}$ were significantly lower in the failed group compared to the success group pre weaning trial and during weaning trial. While $\mathrm{PA}-\mathrm{aO}_{2} \mathrm{G}$ was significantly higher in the failed group.

This finding was in agreement with previous studies by El Maraghi et al., 2014 and Chiwhane \& Diwan, 2016 which showed significant difference between both groups as regards $\mathrm{PO}_{2}$ pre-weaning trial and during weaning trial.

The present study showed that there was significant decrease of both the mean serum magnesium and calcium in failed group compared to the success group.

Disorders of magnesium, calcium are common in patients admitted to ICU. Hypomagnesaemia and hypocalcaemia leads to muscle weakness and respiratory failure, causing difficulty in weaning patient from the ventilator (Safavi \& Honarmand, 2007).

This significant difference in serum magnesium and calcium levels between both studied groups could be attributed to the severity of illness in addition to malnutrition and poor health care in most of patients of the failed group.

Also, anemia is a common co-morbid condition in the ICU, and is found in virtually all critically ill patients (Scheinhorn et al., 2007).

In the present study, the mean hemoglobin level was significantly lower in the failed group compared to the success group, pre weaning and 2 days after weaning trial. Good hemoglobin and Hct levels were found to be associated with weaning success.

Wide debate about the desired hemoglobin level suitable for weaning. Many studies referenced a hemoglobin target of $>10 \mathrm{~g} / \mathrm{dL}$ as weaning guideline (Boles et al., 2007).

In the present study, the CPK-MB level was significantly higher in the failed group after 2 days of weaning trial, mostly due to presence of cardiopulmonary stress compare to success group.

As regards serum interleukin-6 level, the current study showed that there was significant difference between the failed group and success group 2 days after weaning where the serum interleukin-6 level was significantly higher in the failed group.

In the study conducted by Sellarés et al., 2012 on 49 mechanically ventilated patients, showed that change in IL-6 during the SBT in SBT failure was significantly increased compared with the success group.

Also, In the study conducted by Forgiarini et al.,2018 on 54 adult mechanically ventilated patients, IL-6 serum concentrations was significantly higher in patients who failed the SBT than who passed it.

In the present study, the increase of serum IL6 levels was higher in SBT failure compared with success. The possible explanation of this predominance is that weaning process involves cardiopulmonary stress for ventilated patients. As interleukin IL-6 is a major modulator of the stress response, these results may be attributed to the fact that failed patients present 
less favorable reserve conditions for coping with cardiopulmonary stress during the SBT than succeed patients (Aleksandrova, 2012).

As regards CRP the present study showed that there are significantly higher values in the failed group compared to the success group during weaning trial and 2 days after.

This was agreed with the prospective study done by Zaytoun et al., 2014 that included 65 mechanically ventilated adult patients who presented with severe sepsis, where there were significantly higher values in the failed group compared to the success group after $24 \mathrm{~h}$ and after $72 \mathrm{~h}$.

Also in the present study, CRP could predict the outcome of weaning from mechanical ventilation during weaning. CRP at cut-off point $37 \mathrm{mg} / \mathrm{L}$ had corresponding sensitivity of $58.97 \%$ and specificity of $89.47 \%$. .

Luo et al. (2017), showed that CRP level >58 $\mathrm{mg} / \mathrm{L}$ were the independent predictors for weaning failure. They concluded that CRP level of $>58 \mathrm{mg} / \mathrm{L}$ prior to weaning had the best combination of sensitivity, specificity, positive predictive value, and negative predictive value, and diagnostic accuracy for predicting weaning failure.

A strong and independent association of increased CRP and respiratory failure were shown in several studies (Salazar et al., 2014).

CRP is an acute phase reactant, and its plasma level increases four to six hours after a stimulus, Toumpanakis et al., 2015 hypothesized that inspiratory resistive breathing (IRB) results in increased mechanical stress, causing pulmonary inflammation, the intensity of the inflammation is due to cardiovascular stress, induces increased IL-6 levels, which could stimulate increased CRP, reflecting the intensity of the inflammation process.

As regard comparison between the three modes of weaning, the highest success rate was found in the PSV group (76.9\%) while the highest failure rate was in the SIMV group (38.5\%).

In a European multicenter trial, 456 medicalsurgical ICU patients who were clinically ready for weaning and satisfied traditional weaning criteria were studied. The results showed that PSV had the lowest weaning failure rate as compared to SIMV or T-piece ( $23 \%$ vs $42 \%$ and $43 \%$ ) (Brochard et al., 1994).

In the study conducted by Ladeira et al., 2014 to compare pressure-support ventilation with spontaneous breathing through a T-tube for weaning from mechanical ventilation in adults, no clear evidence was found. Matic \& Majeri-Kogler, 2004 compared SBT and PSV as two methods of weaning patients from mechanical ventilation. PSV was the superior method of weaning according to the rate of successful extubation, time of weaning from mechanical ventilation, total time of mechanical ventilation, and length of ICU stay.

The study showed that there was no significant difference on comparing the three modes regarding IL6 and CRP. However, there was a significant difference between the three weaning modes as regards $\mathrm{PO}_{2}, \mathrm{PaO}_{2} / \mathrm{FIO}_{2}$, and $\mathrm{PA}-\mathrm{a} \mathrm{O}_{2}$ gradient measured 2 days after weaning trials. It was found that there was a significant difference when comparing PSV with SIMV groups and SIMV with Once t- piece groups.

So, $\mathrm{PO}_{2}, \quad \mathrm{PaO}_{2} / \mathrm{FIO}_{2}$ ratio and PA-a $\mathrm{O}_{2}$ gradient can be used to compare weaning modes and the lowest value was in the SIMV group.

\section{Conclusion}

It was concluded that patients who failed the SBT presented higher inflammatory parameters than those who passed. CRP could predict the outcome of weaning from mechanical ventilation.

The highest success rate was in the PSV group while the highest failure rate was in SIMV group.

Temperature, RR, RSBI, Minute ventilation, PO2, $\mathrm{PaO} 2 / \mathrm{FIO} 2$ ratio, $\mathrm{PA}-\mathrm{O} \mathrm{O} 2 \mathrm{G}$ and CRP could predict weaning outcome during weaning trial, while RSBI and RR had the highest sensitivity and specificity.

\section{Recommendations}

Simple, economic and easily accessible markers like CRP and IL6 are important determinants of possible outcomes of a patient from mechanical ventilation.

RSBI and RR had the highest sensitivity and specificity among the predictors in our study so, RSBI and RR should be considered for every patient before weaning.

T-piece trial has to be taken in consideration and implemented if choice is made between it and synchronized intermittent mandatory ventilation or pressure support ventilation as methods of weaning patients from mechanical ventilation, it is easily applicable even by the respiratory therapists and the nurses. In contrast, with synchronized intermittent mandatory ventilation and pressuresupport ventilation, ventilator settings must be adjusted repeatedly.

\section{References}

Ahmed SM., Das B., Nadeem A., et al. (2014): Survival pattern in patients with acute organophosphate poisoning on mechanical ventilation: A retrospective intensive care unit-based study in a tertiary care teaching hospital. Indian journal of anaesthesia, 58:117.

Aleksandrova N P. (2012): Cytokines and resistive breathing. Fiziol Cheloveka. 2012; 38(2):11929.

Bhadra K. \& Suratt B. (2009): Drug-induced lung diseases: A state-of-the-art review. $\mathbf{J}$ of Respiratory Disease, 30 (1): 1-17.

Black S., Kushner I. \& Samols D. (2004): C-reactive protein. The Journal of Biological Chemistry. 279 (47):48487-48490.

Boles JM., Bion J., Connors A., et al. (2007): Weaning from mechanical ventilation. European Respiratory Journal, 29(5), 1033-1056.

Brochard L., Rauiss A. and Benito S. (1994): Comparison of three methods of gradual withdrawal from ventilatory support during 
weaning from mechanical ventilation. Am J RespirCrit Care Med 1994; 150: 896-903.

Chiwhane A. and Diwan S. (2016): Characteristics, outcome of patients on invasive mechanical ventilation: A single center experience from central India. The Egyptian Journal of Critical Care Medicine, 4(3), 113-118.

El Maraghi S., Hosny M., Samir M.,et al. (2014): Usage of B-type natriuretic peptide for prediction of weaning outcome by spontaneous breathing trial. Egyptian Journal of Chest Diseases and Tuberculosis, 63(3), 671-678.

Epstein S K. (2009): Weaning from ventilatory support. Current opinion in critical care, 15(1), 36-43.

Forgiarini SG., da Rosa DP., Forgiarini LF., et al. (2018): Evaluation of systemic inflammation in patients being weaned from mechanical ventilation. Clinics, 73.

Georgakas I., Boutou AK., Pitsiou G., et al. (2018): Central Venous Oxygen Saturation as a Predictor of a Successful Spontaneous Breathing Trial from Mechanical Ventilation: A Prospective, Nested Case-Control Study. The open respiratory medicine journal, 12, 11 .

Goncalves EC., Lago AF., Silva EC., et al. (2017): How Mechanical Ventilation Measurement, Cutoff and Duration Affect Rapid Shallow Breathing Index Accuracy: A Randomized Trial. Journal of clinical medicine research, 9(4), 289.

Kallet RH. (2011): Patient-ventilator interaction during acute lung injury and the role of spontaneous breathing: part 1: respiratory muscle function during critical illness. Respiratory care;56(2):181-189.

Ladeira MT., Vital F M., Andriolo RB., et al. (2014): Pressure support versus T-tube for weaning from mechanical ventilation in adults. Cochrane Database of Systematic Reviews, (5).

Lima EJ. (2013): Respiratory rate as a predictor of weaning failure from mechanical ventilation. RevistaBrasileira de Anestesiologia, 63(1), 712.

Luo Z., Cao Z., Zheng Y., et al. (2017): Value of neutrophil-to-lymphocyte ratio, procalcitonin, and C-reactive protein in help to predict weaning outcome: a prospective, observational cohort study.European Respiratory Journal.50: PA2104.

Mabrouk A A, Mansour O F, El-Aziz A A, et al. (2015): Evaluation of some predictors for successful weaning from mechanical ventilation. Egyptian Journal of Chest Diseases and Tuberculosis. 64, (3), 703-707.

Madani SJ.,Saghafinia M., Nezhad HS., et al. (2013): Validity of integrative weaning index of discontinuation from mechanical ventilation in iranian ICUs. Thrita, 2(4), 62-8.

Matic I. and Majeric-Kogler V. (2004): Comparison of pressure support and T-tube weaning from mechanical ventilation: randomized prospective study. Croat Med J.45:162-166.

Monaco F., Drummond GB., Ramsay P., et al. (2010): Do simple ventilation and gas exchange measurements predict early successful weaning from respiratory support in unselected general intensive care patients? British journal of anaesthesia, 105(3), 326333.

Min Y G, Ahn J H, Chan Y C, et al. (2011): Prediction of prognosis in acute paraquat poisoning using severity scoring system in emergency department. Clinical toxicology, 49(9), 840845.

Moçin Ö Y, Karakurt Z, Şen E, et al. (2013): Serum creatinine and weaning in patients with chronic obstructive pulmonary disease: Multicenter pilot study. Journal of Palliative Care \& Medicine, 3(2), 143-150.

Nazima SN., Bashir Y., Nabi S., et al. (2018): Intensive care management of organophosphorus poisoning patients: an experience from tertiary care centre. International Journal of Advances in Medicine, 5(2), 257-264.

Okamoto K., Sato T. and Morioka T. (1990): Airway occlusion pressure (P 0.1) - A useful predictor for the weaning outcome in patients with acute respiratory failure-. Journal of anesthesia, 4(2), 95-101.

Routsi C., Papas V. \& Zakynthinos S. (2013): Neurormonal and inflammatory responses during weaning trials from mechanical ventilation. OA Critical Care, 01; 1(2):13.

Safavi M. \& Honarmand A. (2007): Admissionhypomagnesemia-impact on mortality or morbidity in critically ill patients. Middle East J Anaesthesiol, 19, pp. 645-660.

Salazar J., Martínez MS., Chávez M., et al. (2014): Creactive protein: clinical and epidemiological perspectives. Cardiol Res Pract.; 605810.

Scheinhorn DJ., Hassenpflug MS., Votto JJ., et al. (2007): Post-ICU mechanical ventilation at 23 long-term care hospitals: a multicenter outcomes study. Chest, 131(1), 85-93.

Sellares J., Loureiro H., Ferrer M., et al. (2012): The effect of spontaneous breathing on systemic interleukin-6 during ventilator weaning. EurRespir J; 39 (3): 654-60.

Spadaro S., Grasso S., Mauri T., et al. (2016): Can diaphragmatic ultrasonography performed during the $\mathrm{T}$-tube trial predict weaning failure? The role of diaphragmatic rapid shallow breathing index. Critical Care, 20(1), 305.

Toumpanakis D., Noussia O., Sigala I.,et al. (2015): Inspiratory resistive breathing induces MMP-9 and MMP-12 expression in the lung. Am $\mathbf{J}$ Physiol Lung Cell Mol Physiol; 308((7)):L683-92.

Verona C., Hackenhaar FS., Teixeira C., et al. (2015): Blood markers of oxidative stress predict weaning failure from mechanical ventilation. 
Journal of Cellular and Molecular Medicine. 19 (6): $1253-1261$.

Yoo JW., Lee SJ., Lee JD., et al. (2018): Comparison of clinical utility between diaphragm excursion and thickening change using ultrasonography to predict extubation success.
The Korean journal of internal medicine, 33(2), 331.

ZaytounT., Mahrous AA., Dawood A., et al. (2014): Chromogranin A ( $\mathrm{CgA})$ as a marker of weaning in mechanically ventilated critically ill septic patients. Egyptian Journal of Chest Diseases and Tuberculosis, 63(4), 955-961.

الملخص العريجى

تقييم بعض متنبئات الفطام من التنفس الصناعي لمرضى التسمم الحاد بمركز علاج التسمم بمستشفيات جامعة عين شمس

أسماء أحمد فوزي عساف و هبة محمد حلاوة و جيهان بشرى عزب و أحمد فضل الله يونس ا و جمال الدين محمد أحمد عليץ

الخلفية :هناك المئات من المواد السامة المعروفة التي تسبب تأثيراً مباشراً علي الجهاز التنفسي مما يتطلب وضع المريض على جهاز التنفس الصناعي

• ويعد التنفس الصناعي تدخل منقذ للحياة، وعملية الفطام قد تسبب جهدا شديدا علي عضلات التنفس لذلك يمكن توقع إنتاج السيتوكينات الالتهاب أثناء

عملية الفطام مثل مستوى انترلوكينج وبروتين سي التفاعلي.

الهدف من الدراسة : تقييم دور مستوى انترلوكينج وبروتين سي التفاعلي كمتنبئات لعملية الفطام من التنفس الصناعي لمرضى التسمم الحاد بمركز

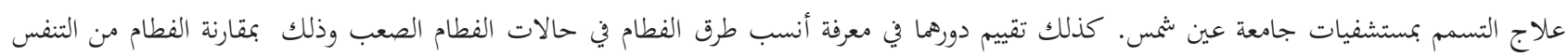

الصناعي بطريقة التنفس الصناعي بالضغط الداعم ،التنفس الصناعي المتزامن المتقطع إلالزامي أو الفطام بمحاولة التنفس الذاتي مرة واحدة يوميا.

المرضى وطريقة البحث: لهذا الغرض قمنا بدراسة مه مريضًا يعانونون من التسمم الحاد وتم وضعهم علي جهاز التنفس الصناعي ، بوحدة العناية

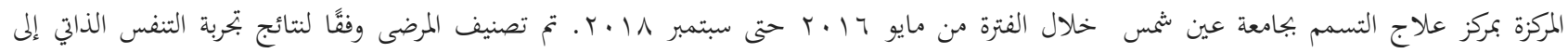

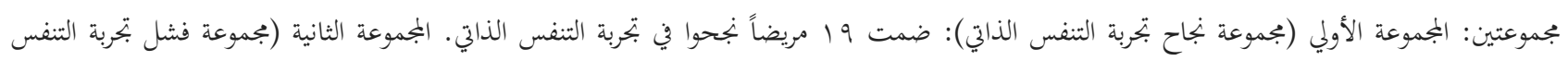
الذاتي): ضمت وسريضا فشلوا في بتربة التنفس الذاتي الذي تم تقسيمه إلى ثلاث مجموعات وفقا لطريقة الفطام: المجموعة (1) مجموعة التنفس الصناعي بالضغط الداعم ، المجموعة (r) مجموعة التنفس الصناعي المتزامن المتقطع إلالزامي ومجموعة (r)بجموعة محاولة التنفس الذاتي مرة واحدة يوميا.، كل بجموعة

النتائج: فيما يتعلق بمستوى الإنترلوكين -7، أظهرت الدراسة أنه يوجد فروق ذات دلالة إحصائية بين المجموعتين ، بعد يومين من بتربة الفطام

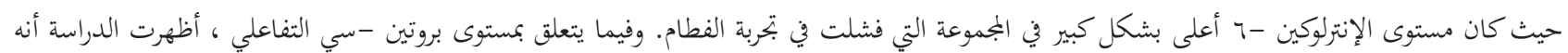

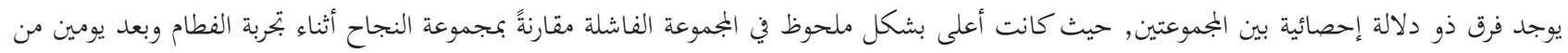

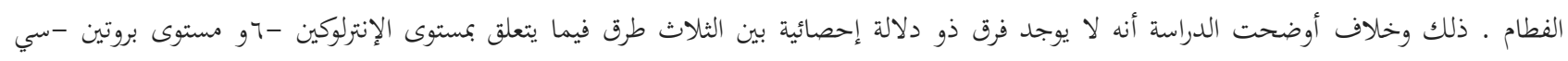
التفاعلي.

الحلاصة: خلصت الدراسة أن مؤشرات الالتهاب كانت اعلي في المجموعة الفاشلة مقارنة بمجموعة النجاح أثناء عملية الفطام وان مستوى

بروتين - سي التفاعلي يمكن أن يتوقع نتائج الفطام.

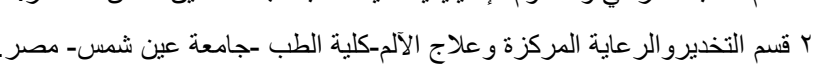

